\title{
Arqueología y Turismo: dos formas de viaje para una misma mirada
}

\section{Archeology and Tourism: two ways of traveling, the same sight}

\author{
Maximiliano Emanuel Korstanje (KORSTANJE, M. E.) * e \\ Lourdes Cisneros-Mustelier (CISNEROS-MUSTELIER, L.) ${ }^{* *}$
}

\begin{abstract}
RESUMEN - Aun cuando el turista y el arqueólogo partan de diferentes plataformas, se encuentran en una misma perspectiva. En este ensayo, se sustenta la tesis que el arqueólogo es un turista solapado. Particularmente, existen diferencias entre ambos, empero poco se ha escrito sobre lo que arqueología y turismo tienen en común. En tal contexto, se cree que el eje de la discusión debe cambiarse para comprender como ambos operan con iguales objetivos. La paradoja es que el turismo "moderno" y la arqueología comparten la misma necesidad de apropiación visual como la búsqueda de la verdad. Pero estos valores fundadores han sido otorgados por el pensamiento científico. El término turismo moderno, se refiriere al turismo capitalizado y no a otras formas de recreación o turismo antiguo. Ello sugiere que se deben resignificar los prejuicios de que el turismo se ocupa de maneras superficiales de consumo, mientras la arqueología es todo lo objetiva que pudiera.
\end{abstract}

Palabras claves: Turismo; Arqueología; Apropiación; Viajes.

ABSTRACT - Although archeology and tourism are based in diverse paradigms, both share the same cultural value. This essay review rests on the thesis that archeologists are mystery tourists. Particularly, there are substantial differences in how archeology portrays the other, but at the bottom, much in common has archeology with tourism. The axis of discussion should be turned to think tourism and archeology as inextricably intertwined. The paradox is that "modern" tourism and archeology share the same need of visual expropriation that leads to the discovery of the truth. These founding values were conferred by scientific thought. The term "modern" tourism refers to capitalized tourism and not other forms of leisure and old tourism. That suggests a re-signification to the prejudice to point out tourism is a naive activity aimed at recreating lay people while archeology keeps an objective gaze.

Key words: Tourism; Archeology; Appropriation; Trips.

\footnotetext{
* Ph D. Social Psychology (Universidad de Moron). Editor en Jefe de las revistas International Journal of Safety and Security in Tourism/Hospitality e International Journal of Cyber Warfare and Terrorism. Revisor de 35 revistas científicas arbitradas. Ha sido nominado a 5 doctorados honoris causa por su contribución al turismo y estudios sobre el terrorismo. Es miembro de la International Society for Philosophers, Sheffield Reino Unido y el Tourism Crisis Management Institute de la Universidad de Florida, Estados Unidos. Docente Investigador (Universidad de Palermo, Argentina). Dirección: Larrea 1079, CP 1414. Buenos Aires, Argentina. Teléfono: 001154 4964-4600. E-mail: maxikorstanje@arnet.com.ar

** Licenciada en Economía (Universidad de Oriente, Cuba); Máster en Gestión Pública de Turismo: Competitividad y Sostenibilidad (Universidad Internacional de Andalucía, España); Máster en Economía (Universidad de Valencia, España); Ph D. en Ciencias Económicas (Universidad de la Habana, Cuba). Decana de La Facultat de Turismo. (Universidad de la Habana, Cuba). Autora de diferentes artículos publicados en revistas científicas. Ponente en eventos nacionales e internacionales. Miembro de la Comisión Nacional de la Carrera de Turismo y de la ANEC. Área de investigación: gestión de los costos y la calidad en la restauración hotelera. Consultora en entidades hoteleras. Dirección: Calle San Lázaro Esq. L. Vedado/Plaza de la Revoluciòn. CP: 10400 - La Habana - Cuba. E-mail: decanato@ @tur.uh.cu
} 


\section{INTRODUCCIÓN}

Como disciplina científica la arqueología ha contribuido en forma tangencial aunque no por eso menos importante, al fortalecimiento del turismo en tanto sector predominante de la economía (ROBB, 1998; ROWAN; BARAM, 2004). Muchos sitios que en el pasado han sido descubrimientos o yacimientos arqueológicos, hoy se posicionan como reconocidos centros de atracción turística, abiertos a miles de visitantes cada año. No obstante, la sociología clásica sugiere que los saberes arqueológicos toman canales totalmente opuestos a las formas de percepción creadas por el turismo (DUKE, 2007). En este ensayo, se discute la dicotomía entre la orientación que una persona tiene frente a un objeto, la percepción y la imaginación. La diferencia epistemológica substancial sobre el cual se centra la discusión es la siguiente.

Cuando un turista visita un centro arqueológico se encuentra frente a muchas piezas, objetos e instrumentos que pertenecieron a otras culturas y que por eso despiertan una curiosidad inusitada para él. Empero, lejos de imaginarse la situación, el turista queda sujeto a una "narrativa" construida por el experto (RUBIO, 2006). En este caso, el guía que transmite en forma sumariada los avances científicos en la materia de los últimos años (COHEN, 1985; REISINGER; STEINER, 2006). El turista, al tratarse de un lego, parece no comprender la complejidad del léxico científico sino a través de un mediador. Por el contrario, el arqueólogo cuando da por vez primera con el objeto que busca (o que encuentra) no tiene una significación cerrada del mismo. No solo no sabe la función del objeto dentro de la sociedad que lo fabricaba, sino su sentido simbólico. Por ese motivo, el arqueólogo debe imaginar, enlazando las diferentes hipótesis a la evidencia histórica ya certificada, validada y documentada por medio del archivo. Las diversas formas de construir el mundo en un arqueólogo y un turista se remiten a diferentes matrices culturales que le dan origen y sustento. Descubrir cuales son esas matrices, como se han introducido en el mundo capitalista, y ver que tal vez no sean tan diferentes, es el objetivo central del presente trabajo. 


\section{ARQUEOLOGÍA Y ANTROPOLOGÍA EN SUS COMIENZOS.}

La historia demuestra que no hubo una disciplina más crítica pero a la vez complaciente con el colonialismo como la antropología y la etnología (KORSTANJE, 2012). Los primeros antropólogos de campo partían de la idea que las culturas no occidentales estaban en vías de desaparecer (HARRIS, 2001). Su misión como científicos no estaba solamente en darle nomenclatura a los objetos o costumbres observadas, sino en almacenar la mayor cantidad de datos posibles sobre todas las culturas que se encontraran dando por hecho que su extinción era cuestión de Tiempo. Muchos gobernadores u oficiales militares apelaban a leer los diarios y las etnografías de los antropólogos de campo para saber como conducirse, disciplinar y adoctrinar a los “salvajes” (STOCKING, 1968; GEERTZ, 1992).

En ese contexto, hasta el proceso de descolonización, y el advenimiento estructuralista, muchos estudiosos coincidieron en señalar que entre la antropología y el colonialismo existió cierta complacencia y complicidad (CLIFFORD, 1988; ERIKSEN; NIELSEN, 2001; COOPER, 2005). No obstante, al mismo tiempo no ha habido otra disciplina que haya estudiado con mayor rigurosidad el rol del etnocentrismo, la alienación y las teorías evolucionistas como la antropología (CLIFFORD; MARKUS, 1986; CLIFFORD, 1999).

Desde sus inicios, la misma no solo estuvo enraizada en la historia sino que compartía la idea de una evolución siempre lineal unida a las formas productivas de cada sociedad (BOAS, 1904). Con el paso del tiempo, una sociedad agraria se vería reemplazada por una capitalista. Dicha doctrina abrió las puertas para el fortalecimiento de una forma paternalista de protección que desembocó en la institucionalización del museo. La identidad de un pueblo próximo a desaparecer fue el segundo gran elemento constitutivo del trabajo de campo. Metodológicamente, la antropología, la etnología y sobre todo la arqueología inauguran la necesidad de abandonar el gabinete para adentrarse en el mundo del nativo. Específicamente, se parte del supuesto que ese "ethos interpretativo" no podía ser decodificado si el investigador se mantenía en su sociedad de origen. Para vencer los prejuicios, el investigador debía desplazarse y observar in situ las prácticas de los aborígenes manteniendo una reflexivilidad que le permitiera llegar a los datos del informante clave con mayor felicidad y objetividad 
(GUBER, 2001). El arqueólogo Matt Edgeworth (2006) entiende que la disciplina arqueológica se ha presentado históricamente como experta y analista en como los pueblos producen su cultura. Lo que olvida, es que ella misma es el resultado de una forma acabada de cultura que la determina con sus propios estereotipos y prejuicios. Lejos de alcanzar la objetividad deseada, la arqueología no representa un encuentro con el otro, sino que a través del otro uno se encuentra asimismo (EDGEWORTH, 2006a).

No obstante, la posición entre observador y observado en la actualidad ha cambiado a puntos radicales en donde ya no existe diferencia entre uno y otro. Hoy el "nativo" maneja no solo la misma tecnología que el etnólogo, sino sus mismas vestimentas, e incluso puede indagar cuestiones esenciales de su trabajo etnográfico (GUBER, 2001). En este sentido, Marc Augé (1998) sugiere que en toda sociedad coexiste un movimiento tendiente a "la historia pasada" preocupado por la tradición y otro a "la historia futura" orientado hacia lo que va a venir. La modernidad actúa reduciendo las lejanías, los espacios y modificando las formas de alteridad. El otro, ya no es un ser "lejano, exótico y diferente", sino que se encuentra - real o imaginariamente - cercano integrado a un mundo más amplio. Por otro lado, la (post)modernidad "crea pasado inmediato" en forma desenfrenada; en otras palabras, todos los días se viven acontecimientos pasados e históricos que desdibujan la línea divisoria entre actualidad e historia. Todo viaje etnográfico apela a un descubrimiento que parte de la imaginación (AUGÉ, 1998). Lo que sin lugar a dudas sugiere el "viaje antropológico" es una necesidad de descubrimiento, de encuentro con un otro sedimentado en la confianza y el tiempo de estancia. El etnólogo, a diferencia de un turista, atraviesa diversos conflictos existenciales para unirse con el otro en uno sólo o fallar en su empresa y volver a su destino. Por ese motivo, el choque cultural es imposible en el turismo y sólo puede darse por medio del conflicto (INGOLD, 2000; 2011; IRWING, 2007).

\section{EL PROYECTO DE CLASIFICACIÓN CIENTÍFICO}

En uno de los trabajos seminales en materia de colonialismo, Mary L. Pratt (2011) esboza una crítica al sistema discursivo de la literatura (y de la ciencia) que es 
pertinente discutir en este trabajo. Pratt (2011) reconoce que la hegemonía imperial nunca es aceptada en forma univoca. El grupo dominado aporta parte de sus expresiones culturales e interpreta a favor del dominador naturalizando su supuesta superioridad. Estos encuentros simbólicos pueden florecer en "zonas de contacto" donde se dan verdaderos procesos de transculturación. Si bien la ideología imperial es elaborada fuera de las comunidades periféricas, la realidad es que su aceptación depende de muchos factores.

No obstante, el imperialismo europeo no hubiese sido posible sin dos mecanismos ideológicos significativos. Uno fue el interés científico por la creación de un modelo clasificatorio que permitió la introducción de una "conciencia planetaria". Gracias a este modelo, los europeos se lanzaron a los mares con el fin de explorar los límites interiores de lo que concebían como un sistema total mundial. El segundo instrumento se refiere a la literatura de viajes, la cual por su parte ha despertado un romanticismo especial que por un lado cuestionaba en forma directa las prácticas colonialistas, y sus injusticias, como la esclavitud o la violencia física, pero por el otro dejaba fuera de discusión otros valores importantes como la superioridad blanca sobre otros grupos humanos (PRATT, 2011). El mérito de Pratt (2011) consiste en denunciar la ambigüedad de la literatura de viajes, la cual por un lado abogaba por un mensaje explícito, mientras en el seno de su narrativa enfatizaba en un paisaje desigual, funcional a la superioridad del orden imperial.

El "ojo imperial” se presenta como Adán en el paraíso, con posibilidades de disfrutar y ver todo a su paso, sin que nada lo pueda detener. Este ojo no solo escudriña y controla sino que además construye paisajes simbólicos donde la dominación europea nunca es cuestionada. Esta clase de literatura dice más por lo que esconde que por lo que puede denunciar. En la mayoría de los estudios de caso, novelas y textos analizados por Pratt (2011) el nativo no posee nombre, ni historia, mucho menos una identidad propia que lo defina fuera de lo que es su vínculo con el europeo. Dos eventos han sido de vital importancia para marcar a fuego la historia del colonialismo. Según Pratt (2011), en 1735 Carl Linneo publica su trabajo Sistema Naturae en donde establece la necesidad de clasificar en forma sistemáticas diversas plantas, especies herbáceas y vegetales tanto europeos como extra europeos. En forma casi paralela, parte una expedición naviera con rumbo a América del Sur conocida como "expedición 
Condamine" para poder hacer mediciones más precisas sobre la forma de la tierra. Si bien esta última expedición ha sido un fracaso desde lo científico, produjo una serie de documentos que alimentaron el interés de los europeos por un nuevo género, las experiencias vertidas en los relatos de viajes. Estos textos comenzaban dando una ubicación geográfica más o menos precisa del viaje y narraban (siguiendo el sistema clasificatorio) las diversas costumbres, culturas y personas que conocían durante la travesía. Pronto este tipo de relatos comenzaron a ser funcionales a la lógica burguesa de acumulación europea que estaba transformando las antiguas instituciones medievales. La retórica de los viajes, desde sus inicios, apeló a un sentimiento sensacionalista donde el otro "no occidental" quedaba subordinado (innominado) a una matriz cultural imperial que le daba a su vez sentido, a lo observado (PRATT, 2011; .

El colonialismo no podría haber sido posible sin esta "conciencia planetaria" propuesta por la ciencia de ese entonces. La táctica imperial aludía a la posibilidad de crear ciertos estereotipos, marcadores y signos que son atribuibles a otros, pero en ese mismo acto, el centro quedaba intacto de ser marcado. A los peligros que deben atravesar los "valientes europeos" se le combinaban toda una serie de maravillas que despertaban no solo la curiosidad sino la ambición de comerciantes y colonos de todo tipo, color y clase. La necesidad de una historia natural que diera sentido a la cultura europea pero a la vez redujera el conflicto, la disidencia a su mínima expresión, fueron los motivos para la creación de la literatura de viajes y las posteriores ciencias positivas derivadas de ésta.

\section{EL ACTO DE DESCUBRIMIENTO ARQUEOLÓGICO}

Michel Foucault se consideraba "un arqueólogo" aun cuando no tuviese la acorde titulación como tal, por la función que ejercía en descubrir aquellos discursos que habían sido enterrados por acción de la hegemonía (FOUCAULT, 1988). Toda estructura política del saber se corresponde con un corpus teórico consensuado, validado por la historia y la ley, y otro silenciado, desterrado de las fauces científicas. Este proceso lejos de ser estático se encuentra en constante negociación. Descubrir 
trabajando sobre los archivos aquellas voces ignoradas por la sapiencia oficial representa un acto de descubrimiento (FOUCAULT, 1988; 1997).

El aspecto central ignorado por Foucault ha sido sin lugar a dudas la interpretación que se le da al acto de descubrimiento. Gilbert Ryle (1963) sostenía que una estructura no se construye por lo que la define materialmente, sino por el sentido otorgado sobre ella. Una universidad no solamente es la cantidad de programas o el edificio, sino un sinnúmero de interacciones humanas que hacen un todo institucional. La interpretación de esas relaciones determina su función social (RYLE, 1963). El acto de descubrir sólo adquiere sentido por la confianza que adquiere el descubrimiento. Los círculos científicos pueden sepultar o valorizar una teoría dependiendo del grado de confianza que ella genera, aun cuando descanse sobre una gran falacia (SOKAL; BRICMONT, 1998).

Siguiendo este argumento, Richard Schmitt (1995) sugiere que es un error pensar a la persona como entidad autónoma, productora de sentido y de experiencia. Los sujetos eligen tanto trabajar en relación con, como separarse para maximizar sus ganancias en forma solitaria. Lejos de lo que supone el imaginario colectivo, el científico necesita de la confianza de otros para poder validar los hallazgos de su trabajo. La construcción de paradigmas no solo refleja formas estructuras para comprender la realidad, sino además intentos voluntarios de sociabilidad entre los círculos científicos. Sin embargo, al hacer eso, se excluyen a otras voces y opiniones con el peso suficiente para cambiar de pensamiento (SCHMITT, 1995).

Merleau Ponty (1964) lucha insistentemente contra la epistemología de la ciencia que propone una persona ajena, objetiva al mundo circundante, que puede abstraerse del objeto que estudia hasta poder trazar una muralla entre quien observa, y el objeto observado (MERLEAU-PONTY, 1964). Paul Riccoeur (1977), reconoce al igual que Ponty, que la frontera divisoria proclamada por el racionalismo es imposible, pero apuesta a la teoría de la representación como una manera viva de imaginar. A diferencia de la estructura que es fija, la imaginación permite una actualización constante de las instituciones humanas. Su razón de ser descansa en la ausencia del código normativo (RICCOEUR, 1977). La imaginación parte por la falta de norma clara, de código pero a la vez se reinventa asimisma para conferir un sentido a esa ausencia. 
Lucas (2007) advierte que el investigador va hacia el objeto pero una vez que lo encuentra queda condicionado por éste. Si se define a la práctica arqueológica por abrir un solo sentido, sus hallazgos permiten tomar en sí miles de conclusiones, muchas otras variantes interpretativas que hace del encuentro un re-encuentro (LUCAS, 2001). El modelo experimental positivista de la ciencia trabaja en estadios replicables, donde el observador puede tener acceso al dato pues se encuentra disponible en tiempo presente. Empero, ni los historiadores menos los arqueólogos corren la misma suerte. Los datos deben ser replicados sin que el observador pueda recabar la información por medio de sus sentidos. Al no tener una norma fija, el arqueólogo debe imaginar para luego validar con la evidencia existente. No obstante, aunque se sigan reglas pautadas, ese acto de descubrimiento no esconde su matiz político. El objeto descubierto tiene una doble pertenencia, por un lado habla de una civilización extinta que ya no puede decir nada, pero también se refiere a una expropiación cultural y una nueva refundación acorde a propios intereses culturales (EDGEWORTH, 2006a; 2006b). Los rituales, procesos de descubrimiento en la disciplina obedecen a prácticas establecidas normativamente que trazan fronteras epistemológicas con otras disciplinas. El poder interpretativo subyace sobre los discursos previamente consensuados y aprobados de lo que significa ser "un buen arqueólogo" (WILMORE, 2006).

Siguiendo este argumento, Van Reybrouck e Jacobs (2006) plantean un dilema epistemológico alrededor de la excavación arqueológica. Se parte del supuesto que el arqueólogo es un científico, un rescatista que va tras los pasos del pasado. Su misión parece clara a grandes rasgos, salvar los fragmentos de civilizaciones pasadas para ser conservadas y admiradas por los ojos modernos. No obstante, los autores sugieren que el hecho por medio del cual se puede legitimar el hallazgo arqueológico no es consecuencia de la investigación sino de la doble hermética profesional. Los grupos humanos de la pre-históricos no adquieren una entidad sin una definición previa de lo que es la prehistoria para los eruditos.. Ello sugiere que el hallazgo (hecho) queda sujeto a la interpretación que se hace de la pre-historia. Lo curioso, es que la práctica que define el descubrimiento se encuentra enraizada en tiempo presente. Por lo tanto, lo que entra en juego en una excavación no es "un descubrimiento objetivo", como tampoco una construcción negociada. Lo que se encuentra en juego es un doble condicionamiento entre el hecho y el actor social. Un aspecto fundamental del 
descubrimiento es que se hace por medio de un instrumento, nunca con las propias manos. Las personas que participan en la excavación son instrumentalmente entrenadas para descubrir empleando la tecnología disponible.

Por último, el tema ético-moral también debe ponerse en discusión. Los etnólogos para entrar al campo de trabajo deben llenar una serie de formularios y autorizaciones para cumplir los requisitos del estado, pero también deben pedir una autorización a la comunidad estudiada. Deben negociar con ellos que se va a publicar, en que contextos y bajo cuales condiciones. A diferencia de la etnología, la arqueología sólo requiere de los permisos conferidos por el estado nacional, pues el grupo humano estudiado ya no existe. No solo el arqueólogo no es confrontado por el otro, lo cual lo asemeja bastante al turista, sino que además desacredita la autoridad legal de los habitantes locales, descendientes o no de la civilización extinta. ¿Podemos considerar al descubrimiento arqueológico un robo?. En materia legal se han puesto en movimiento muchas legislaciones a nivel nacional, continental y mundial con el fin de frenar el tráfico de objetos, pero no se ha podido resolver la exclusión de los actores locales en la negociación por las excavaciones. Poco a poco la tendencia parece querer revertirse. En la actualidad, algunas pocas piezas de museo están siendo repatriadas a las comunidades desde donde fueron extraídas.

\section{EL TURISMO}

El objetivo de la presente sección no es discutir la historia del turismo, sino las formas en las cuales la literatura sociológica lo ha re-retratado. Uno de los primeros detractores del turismo fue Urbain (1989), quien sostenía que el turista lejos de encontrarse comprometido con los lugares que visita, se abandona a los placeres hedonistas de la vida. Por ende, su descubrimiento siempre queda obstruido por una falta evidente de crítica. El viaje turístico abre una consciencia paisajística que es alienadora y cosificadora. Otro académico que fue en la misma vía es el antropólogo Dean Maccannell (2003) quien retoma el legado marxista y estructuralista para proponer que el turismo es el totemismo por otras vías. En tanto que ávido lector de Levi-Strauss, el sociólogo americano reconoce su exclamación cuando afirma que la 
sociedad industrial está vaciada de "totemnismo". Las sociedades primitivas poseen un fuerte valor simbólico y apego a su tradición. Por ende, el grado de fragmentación es débil. Las sociedades industriales han generado un desapego producido por el declive de la confianza. Eso he suscitado que el espacio del totemnismo sea ocupado por formas estereotipadas de consumo, en donde los ciudadanos vuelcan todas sus expectativas, el turismo. Determinado el hábito turístico desde la estructura, el sujeto no puede acceder a formas de viaje diferentes de las estipuladas. En el planeta, se estipula que hay culturas fuertes y avasallantes como débiles. Las primeras tarde o temprano se comerán a las segundas (MACCANNELL, 1988; 2003; 2007). El encuentro por ende no es un espacio de reflexión y reflexivilidad sino un acto político de sumisión. Debe reconocerse que el viaje turístico ya no representa ningún tipo de descubrimiento porque anula al otro a los deseos del turista-consumidor. Su ética queda reducida a lo que puede el sistema explotar de él. Al perderse el interés por el otro, la motivación se pierde en una falsa experiencia en vistas de una falsa caridad. Porque no existe confianza en el prójimo se acelera una suerte de obsesión por la autenticidad (MACCANNELL, 2011).

A diferencia de Maccannell, John Urry (2002) postula la existencia de la "mirada turística" (tourist-gaze), la cual opera en similitud al discurso médico. El sociólogo inglés no ve la necesidad de comparar dos opuestos ya que no tiene influencia directa, como si lo hace el estructuralismo francés. Si bien ambos parten de una noción negativa sobre el turismo, la forma en que cada uno resuelve la interacción entre norma y agencia varían sustancialmente. Ciertamente, Urry enfatiza en la tesis del consumo visual, empero no fuerza la lógica de la autenticidad. Los paisajes que disfruta una persona cuando viaja se construyen a través de una matriz cultural sistematizada, pero que lejos de ser univoca o estructural se constituye desde varias perspectivas subjetivas. El objetivo del viaje y las experiencias juegan un rol primordial en la configuración del ethos moderno. La devoción occidental por lo visual tiende a hacer extraordinario el espacio común. La mirada turística (que es sólo moderna) implica no solo una forma de socialización específica, sino también de expropiación. La evasión para Urry (1995; 2002) es una necesidad que se hace masiva e impuesta por la modernidad para que el trabajador pueda replicar los valores del trabajo a otras esferas del consumo humano. El turismo comoditiza a las personas y a sus afiliaciones territoriales con el fin de transformarlos en productos, vendible y consumibles acorde a los términos del mercado. 
La movilidad en tanto producto cultural se sustenta en la literatura de viajes uniendo imaginarios monopolizados por el estado nacional. A diferencia de otras épocas, la economía moderna produce signos y valores agregados, subordinando todas sus instituciones y desdibujando los alcances del espacio cartesiano. Siguiendo este desarrollo, los marcadores de lugares que caracterizaban al espacio premoderno, en donde se llevaban a cabo los rituales de la vida cotidiana, se han esfumado dando como resultado un lugar que en su abstracción no busca ser vivido sino apreciado. Tomando las influencias nietzscheanas, la re-subjetivización propia de este proceso ha llevado a una dicotomización de las estructuras vigentes que obligan a redefinir el papel del sujeto como entidad inserta en el mundo. Las transacciones comerciales, las jerarquías empresariales, las modas se han transformado y se hacen más horizontales que en épocas anteriores. La alegoría ha reemplazado al símbolo en un horizonte a-cronológico que desdibuja la tradición y la historia (LASH; URRY, 1998).

En este mismo sentido, R. Tzanelli (2013) argumenta convincentemente que el turismo y el clima de festividad que éste despierta, se explican por el imperativo alegórico del capital y la consecuente explotación económica. En perspectiva, el turismo recrea "economías" del pensamiento las cuales no solo moldean la nacionalidad, sino también la identidad de las personas. Subordinada al consumo turístico, en la actualidad, la historia valoriza criterios nostálgicos por el pasado. Desde dicho paradigma, Tzanelli explica que la historia queda construida desde el presente con fines puramente comerciales. La idea no es indagar realmente acerca del pasado de un pueblo, sino hacer converger sus valores para que sean atractivos a quienes se apropian de ellos. Se forma, de esta manera, un "carácter nacional" que conecta universalmente a las naciones con consumidores internacionales. El problema en este sentido, es que no existe consenso en señalar si el turismo es producto de la movilidad, o que por medio de una pseudomovilidad, el estado nacional pudo imponer formas económicas sedentarias.

En vistas de ello, Guidotti-Hernández (2011) enfatiza en el carácter ideológico de las historias fabricadas por el turismo. Los hechos históricos tal y cual han sucedido son invisibilizados para crear una imagen que no solo coincide con el estereotipo político del estado nación, sino con el capitalismo. A diferencia de Urry, quien sostiene que en un mundo móvil, Guidotti-Hernández permite comprender que precisamente el estado nacional ha disciplinado a varios grupos seminómadas y los ha homogenizado a 
tal punto de crearles un sentido por el cual deben vivir juntos, la nacionalidad. La violencia explícita ejercida sobre ellos ha salido del debate historiográfico sin ningún tipo de cuestionamiento. A diferencia de otros autores, el turismo para la autora representa el brazo ideológico de las formas de producción post-capitalistas.

Gran parte de la sociología del turismo, iniciada por Maccannell (2003) y Urry (2002), considera que la modernidad actúa en forma ideológica desdibujando el pasado y creando presente inmediato. Ello sugiere que el descubrimiento del otro es inexistente o se da en forma cerrada. No existe manera de interactuar pues la imagen del otro se ha diseñado previamente desde el deseo del turista. Autores como Augé (1998) o Paul Virilio (1991) afirman que el turismo es un viaje a ningún lado, o una travesía que nunca empieza. No obstante, eso parece ser parte del problema que escapa al diagnostico completo.

Mazierska (2013) critica la "postura crítica" sobre el turismo por dos motivos centrales. La supuesta falta de autenticidad que el turista quiere experimentar, y que puede no darse en otros viajeros es falsa. Pensar que el turista es un agente alienado y despersonalizado es un instrumento simbólico para relatarse uno mismo que "nunca será un turista". Pero al desear ser un "autentico viajero", uno mismo se transforma en lo mismo que crítica, un viajero buscando ser diferente. Ello genera un turista autoreflexivo que niega su propia constitución existencial. El segundo problema subyace en la miopía de algunos críticos que no conciben que el turismo no comience cuando el viajero alcanza su destino sino en el momento en el cual el objeto que ha promovido el viaje es despojado de su sentido oculto. Mediante el proceso por el cual el turista descubre, su búsqueda personal culmina. El objeto deseado funciona como válvula catalizadora que da al deseante lo que busca.

A pesar de sus diferencias, la arqueología y el turismo comparten muchas similitudes que ameritan ser discutidas. Lo que en principio puede ser un punto de ruptura entre el turismo y la arqueología, en el fondo dista de serlo. Angela McClanahan (2006) denuncia la pugna existente entre pobladores locales e inversores turísticos en comunidades arqueológicamente ricas. Y al hacerlo, pone al rol del científico bajo el ojo ácido de la crítica. Los turistas llegan, y con ellos todas las supuestas asimetrías materiales y problemas de impacto social, porque los científicos estuvieron primero. Por su parte, Jean Baudrillard (1994) sugiere que la necesidad de coleccionar obedece a 
impulsos internos que llevan a la persona a pensar que puede controlar el mundo circundante. La ilusión de perfeccionismo del coleccionista lo lleva a un tiempo que nunca es real, sino una forma regresiva de infantilismo (BAUDRILLARD, 1994).

Por el contrario, Korstanje (2013) explica que la necesidad occidental de acumulación no solo se observa en las finanzas, sino en todas las esferas de la vida. El museo, pieza indiscutible ideológica del imperialismo, tiene como función central trivializar una historia traumática de expropiación o dolor para una comunidad. En ese proceso, la comunidad recupera un motivo para mantenerse unida. Los procesos de globalización desdibujan las líneas de lealtad de la ciudadanía hacia sus gobernantes a la vez que reduce la confianza en el otro. La museificación recupera la razón de ser del grupo humano, evitando la fragmentación. Por ese motivo, a medida que la sociedad capitalista se expande, mayores son sus obsesiones por la autenticidad. Este argumento lleva a cuestionar, ¿son el turismo y la arqueología formas de regresión?

\section{SIMILITUDES ENTRE TURISMO Y ARQUEOLOGÍA}

En primera instancia, tanto el turismo como la arqueología parten de matrices culturales que le dan sentido e interpretación a los descubrimientos. Estas matrices se encuentran ideológicamente construidas, encuadradas y orientadas a legitimar el mundo del trabajo. Si bien la arqueología debe apelar a una imaginación que al turismo se le entrega como un discurso ya elaborado, lo cierto parece ser que el objeto confiere valor a las experiencias de ambos. Desde diversas perspectivas, el turista como el arqueólogo son buscadores de extraorinariedad y novedad. El éxito o fracaso de una investigación de campo en arqueología no depende más que de cuan novedosa sea la pieza encontrada. Objeto que será rememorado y sacralizado en un museo, a disposición del ojo turístico. En este sentido, la necesidad de clasificación no solo substrae al mundo deseable a objetos controlables, sino que además les confiere un valor. De hecho, como se ha discutido, si el turista retrata el paisaje empleando una tecnología digital, el arqueólogo llega al objeto de igual forma, haciendo mediar un instrumento.

El segundo aspecto en común es la apropiación cultural. Al igual que el turista, el arqueólogo profesional debe reconstruir un pasado del cual poco se sabe. Ninguno de 
los dos han experimentado el evento que rememoran, y al hacerlo hacen de suyos hechos que fueron culturalmente determinados en otro tiempo y espacio. En perspectiva, otorgan al tiempo pasado un interés muy particular que los lleva a la protección y el paternalismo. Los turistas consumen historias de conquistas y de dominación simplemente porque los antropólogos arman los museos conmemorativos.

Por último pero no por eso menos importante, el arqueólogo es un turista encubierto. Como bien dice Thomas Yarrow (2006), los etnólogos reconocen que los arqueólogos tienen ninguna o escaso contacto con los pobladores locales. En parte, porque saben que esa interacción mínima puede apartarlos de su objetivo principal. Sin embargo, no solo por eso el arqueólogo debe ser considerado un turista. Desde la perspectiva del deseo, el arqueólogo deja de buscar cuando lo que observa es suficiente, al igual que otros científicos o cientistas sociales. La reflexivilidad con ese otro nativo es circunstancial o en el peor de los casos instrumental, buscando sacar un rédito específico. La producción de sentido del objeto encontrado, será comercializada por medio de diversos canales, puesto a consideración del turista para seguir alimentando el círculo ideológico occidental. Empero, bajo la lógica del descubrimiento subyace la misma dinámica, la producción y expropiación del sentido. La tecnología de la museificación, precisamente, funciona porque se le confiere al objeto un valor tan grande que lo quita del canal económico de intercambios. En términos de Weiner (1992), pasa de ser un objeto alienable y por comercializable a otro inalienable, vedado para ser comprado o vendido en el mercado formal. Este proceso empuja al turista a aferrarse a la "replica", a la copia que tanto preocupaba a Dean MacCannell (2003; 2007). No es el turismo por sí como piensan muchos sociólogos el que crea la replica para ser consumida, sino la ciencia moderna que hace de lo auténtico, de la verdad, lo explicable su objetivo constante. La paradoja se da porque cada vez que se encuentra lo auténtico, hay algo de inauténtico que se produce. En cada objeto real existe irrealidad. Por ende, ciencia y mercado son dos caras de la misma moneda. La pregunta que inevitablemente esta discusión plantea, es ¿hasta que punto el proyecto de la ciencia ha sido el creador de la mirada turística que tanto crítica Urry?

Quien mejor explica esto en su retrato sobre la literatura francesa es el investigador de la Universidad de Plymouth, Charlie Mansfield (2008), cuya obra “Traversing Paris" se discute a continuación. 


\section{EL RETRATO TURÍSTICO}

Partiendo de una preocupación común a los literatos, Mansfield (2008) se propone examinar la literatura francesa moderna, con el objetivo de redefinir las fronteras epistemológicas del viaje.. Porque la literatura permite dilucidar como se construyen las narrativas de una época, es que admite Mansfield (2008), se puede comprender la relación entre el viaje moderno, la literatura y el capitalismo. Viajar es algo más que moverse, implica una planificación previa. Ese proceso de racionalización implica imaginarse un paisaje social y re-escribirlo en la propia mentalidad. El turismo moderno no solo es heredero de la literatura de viajes, sino que mantiene su propia epistéme del pensamiento científico. Todo debe partir del mismo punto de origen, la exploración. Pero para hacerlo, uno debe abandonar el confort, la seguridad y la comunidad de la propia sociedad. Debido a esa búsqueda de realidad externa, el hombre pone a disposición la técnica. La práctica de retratar los lugares exóticos y de escribir las propias experiencias ha jugado un rol importante como forma técnica de creación de discurso y a la vez de consciencia. La literatura le daba el valor suficiente al espacio no europeo para hacerle creer al viajero que lo que miraba era la realidad. No obstante, ese sentido de realidad no tenía lugar fuera del texto que le dio precedente.

Siguiendo este razonamiento, agrega Mansfield (2008), el género literario de viajes intentaba cerrar la brecha entre la objetividad y la subjetividad. Dicha brecha no tenía sentido sin la presencia del otro no europeo. De otro diferente que en su “diferencia" queda determinado por el retorno así mismo. En otras palabras, partiendo de la base que el otro es una consecuencia de las proyecciones del self, la literatura de viajes permitía redescubrir lo escondido en la sociedad del escritor. Esta misma forma de pensar ha dado origen a muchas disciplinas sociales como la etnología, la antropología y la arqueología.

Mansfield (2008) discute la epistemología moderna sobre la importancia de considerar al turismo una actividad alienadora y superficial. Como sea, viajar por turismo implica re-descubrir lo que está escondido. La abundancia de comida durante los viajes precisamente emula lo que se ha explicado. La comida ejemplifica en la literatura las injusticias sufridas por la clase trabajadora la cual es explotada por el capitalismo moderno. La ciudad no solo es retratada como una jaula, sino que impulsa a 
los viajeros a la búsqueda de una verdad que sólo puede encontrarse en el mundo natural. Ello genera un círculo hermenéutico que culmina en el "terror a la muerte". El marco creado por el retrato da legitimidad a lo que puede observarse. El arquetipo del retrato confiere valor a lo que se observa y le hace creer a otros que la idea debe ser continuada. En el fondo, esta táctica constituye un mecanismo de defensa frente al paso del tiempo. Como consecuencia, el turismo, la literatura y la ciencia parecen perseguir objetivos conjuntos. A la necesidad de controlar los aspectos negativos del ambiente natural se le combina la imperiosa obsesión por volver a una naturaleza prístina y no habitada por el hombre, con el fin último de conseguir la verdad.

\section{CONCLUSIONES}

El criterio de verdad, historia y objetividad ha sido debatido en forma amplia en varios círculos académicos. No es la intención de este trabajo cuestionar que se entiende por verdad ni por historia, sino simplemente trazar las bases epistémicas para comprender porque hoy miles de turistas (lejos de ser personas alienadas) hacen largas filas para entrar a un museo. Entendido éste último como un dispositivo ideológico creado por la ciencia para conferir sentido a los objetos expuestos. Empero ni las interpretaciones, ni los discursos tras los objetos escapan al diseño político de una forma específica de discurso, enraizados en el "ethos capitalista" que hace del control visual una manera de apropiación. La mirada turística (tourist-gaze) busca precisamente objetos que recuerden la superioridad de la técnica científica occidental frente a otras voces no formales de saberes. En segundo lugar, el turista busca la extraordinariedad que sólo puede darle la arqueología. Y lo hace por dos motivos. Como bien sugiere Mansfield (2008), el primero y más poderoso es el intento por controlar el paso del tiempo. El segundo consiste en lo que Pratt (2011) llama el "ojo" imperial que intenta emular el papel de Adán en el paraíso. Quien visualiza ejerce un marcador sobre quien es visualizado, pregunta por un sentido que le es impuesto a quien es observado. En ese acto y es ahí donde reside el poder del observador, nada sabe el observado de quien observa. 
En este sentido, es necesario examinar las similitudes entre el turismo y la arqueología hasta el punto de considerar que ambos persiguen objetivos complementarios. La arqueología intenta rescatar del pasado a grupos humanos que son moldeados según los ojos del presente. Esa apropiación material y simbólica es consumida visualmente por el turismo. Ciertamente no es extraño observar, que ambos están determinados por la búsqueda de la verdad. El arqueólogo es un turista solapado. Por último pero no por ello menos importante, el concepto de autenticidad tal y como fue formulado por MacCannel (2007) no se sustenta por acción ideológica o de diseño como él pretendía, sino por el rol de la ciencia misma. Los objetos arqueológicos distan de tener valor debido a que son "sacralizados", y porque se los excluye del sistema formal económico de intercambio es que se necesita de la copia, de la replica. La dicotomía autenticidad versus falsedad no solo es falsa, sino que parten del mismo interés de la ciencia por la verdad.

\section{REFERENCIAS}

AUGÉ, M. Turismo: el Viaje Imposible. Barcelona: Gedisa, 1998.

BAUDRILLARD, J. The System of Collecting. In: ELSNER, J.; CARDINAL, R. The cultures of Collecting. London: Reaktion Books, 1994, p. 7-24

BOAS, F. The history of anthropology. Science, v. 20, n. 512, 1904, p. 513-524.

CLIFFORD, J.; MARCUS, G. E. (Eds.). Writing Culture: The Poetics and Politics of Ethnography;[experiments in Contemporary Anthropology]. California: University of California Press, 1986.

CLIFFORD, J. The Predicament of Culture: Twenthieth-Century Ethnography, Literature, and Art. Massachusetts: Harvard University Press, 1988.

Itinerarios transculturales. Barcelona: Gedisa, 1999.

COHEN, E. "The tourist guide: The origins, structure and dynamics of a role". Annals of Tourism Research, v. 12, n. 1, 1985, p. 5-29.

COOPER, F. Colonialism in question. Berkeley: University of California Press, 2005.

DUKE, P. G. The tourists gaze, the Cretans glance: archaeology and tourism on a Greek island. California: Left Coast Press, 2007. 
EDGEWORTH, M. "Preface". In: Ethnographies of Arqueological Practices. Edited by Matt Edgeworth. Lanham: Altamira Press, 2006a.

. Multiple Origins, Development, and Potential of Ethnographies of Archeology. In: Ethnographies of Arqueological Practices. Edited by Matt Edgeworth. Lanham: Altamira Press, p. 1-19, 2006b

ERIKSEN, T. H.; NIELSEN, F. S. A history of anthropology. London: Pluto Press, 2001.

FOUCAULT, M. Las palabras y las cosas: una arqueología de las ciencias humanas. Buenos Aires: Siglo XXI, 1988.

La arqueología del saber. Buenos Aires: Siglo XXI, 1997.

GEERTZ, C. La interpretación de las culturas (V. 1). Barcelona: Gedisa, 1992.

GUBER, R. La etnografía: método, campo y reflexividad (V. 11). Buenos Aires: Editorial Norma, 2001.

GUIDOTTI-HERNANDEZ, N. Unspeakable Violence. Remapping Us and Mexican National Imaginaries. North Caroline, Durham: Duke University Press, 2011.

HARRIS, M. The rise of anthropological theory: A history of theories of culture. New York: Rowman Altamira, 2001.

INGOLD, T. The Perception of Enviroment: essays on livelihood, Dwelling and Skill. London: Routledge, 2000.

Routledge, 2011.

Being Alive. Essays on movement, knowledge and description. London:

IRWIN, R. Culture Shocks: negotiating feelings in the field. Anthropology Matters. V. 9, n. 1, 2007, p. 1-11

KORSTANJE, M. Reconsidering cultural tourism: an anthropologist's perspective. Journal of Heritage Tourism, v. 7, n. 2, 2012, p. 179-184.

Guerra y museología: una introducción a la teoría de los museos. Aposta: Revista de ciencias sociales, v. 56, n. 4, 2013, p. 1-30.

LASH, S.; URRY, J. Economías de Signos y Espacios. Sobre el capitalismo de la postorganización. Buenos Aires: Amorrortu Editores, 1998.

VON LINNEO, C. Naturalista sueco, autor de. Systema Naturae, 1735.

LUCAS, G. Critical Approaches to the Fieldwork. Contemporary and historical arqueological Practice. London: Routledge, 2001. 
MANSFIELD, C. Traversing Paris: French Travel Writing practices in the Late Twentieth Century. Saarsbrucken: VDM Verlag, 2008.

MACCANNELL, D. "Turismo e Identidad cultural". En Todorov, Tzvetan. El Cruzamiento entre culturas. Madrid: Ediciones Jucar", 1988

Tourist agency. Tourist studies, v. 1 n. 1, 2001, p. 23-37.

The tourist, a new theory of leisure class. Moia, Spain: Ed. Melusina, 2003.

Lugares de Encuentro Vacío. Barcelona: Melusina Ed, 2007.

The Ethics of Sightseeing. Los Angeles: University of California Press, 2011.

MCCLANAHAN, A. Histories, Identity and owndership: an ethnographic case study in Archeological Heritage Management. In: Ethnographies of Arqueological Practices. Edited by Matt Edgeworth. Lanham,:Altamira Press, 2006, p. 126-136.

MAZIERKA, E. Tourism and Travelling in Jean Luc Godard's Allemagne 90 Neuf zero and Éloge de l'amour. In: Open Roads, Closed Borders: the contemporary French Language Road Movies. GOTT, M.; SCHILT, T. (org.) Chicago: Chicago University Press, 2013, p. 119-136

MERLEAU PONTY, M. Sense and Non-sense. Evanston: Northwestern University Press, 1964.

PRATT, M. L. Ojos Imperiales: literatura de viajes y transculturación. Buenos Aires, FCE, 2011.

REISINGER, Y.; STEINER, C. J. Reconceptualising object authenticity. Annals of tourism research, v. 33, n. 1, 2006, p. 65-86.

RICCOEUR, P. La metáfora Viva. Buenos Aires: Megalópolis, 1977.

ROBB, J. G. Tourism and legends archaeology of Heritage. Annals of tourism research, v. 25, n. 3, 1998, p. 579-596.

ROWAN, Y. M.; BARAM, U. (Eds.). Marketing heritage: Archaeology and the consumption of the past. New York: Rowman Altamira, 2001.

RUBIO, S. P. La ciudad fingida. Representaciones y memorias de la Barcelona turística. Pasos. Revista de turismo y patrimonio cultural, v. 4, n. 1, 2006, p. 13-28.

RYLE, G. The Concept of Mind. Middlesex: Penguin Books, 1963.

SCHMITT, R. Beyond Separateness. Boulder: Westview, 1995. 
SOKAL, A. D.; BRICMONT, J. Intellectual impostures: Postmodern philosophers' abuse of science (p. xiii-xiii). London: Profile Books, 1998.

STOCKING, G. W. Race, culture, and evolution: Essays in the history of anthropology. Chicago: University of Chicago Press, 1968.

URBAIN, J. D. The tourist adventure and his images. Annals of tourism research, v. 16, n. 1, 1989, p. 106-118.

URRY, J. Consuming Places. London: Routledge, 1995

The Tourist Gaze. London: Sage, 2002

TZANELLI, R. Olympic Ceremonialism and the Performance of National Character, from London 2012 to Rio 2016. Basingstoke: Palgrave-Macmillan, 2013

Van REYBROUCK; JACOBS, D. The Mutual Constitution of Natural and Social Identities during archaeological fieldwork. In: Ethnographies of Arqueological Practices. Edited by Matt Edgeworth. Lanham: Altamira Press, 2006, p. 33-44.

VIRILIO, P. La Inseguridad del Territorio. Buenos Aires: La Marca, 1991

WEINER, A. B. Inalienable possessions: The paradox of keeping-while-giving. Berkeley: University of California Press, 1992.

WILMORE, M. Landscapes of Diciplainary Power: an ethnography of excavation and survey at Leskernick. In: Ethnographies of Arqueological Practices. Edited by Matt Edgeworth. Lanham: Altamira Press, 2006, p. 114-125.

YARROW T. Sites of Knowledge: different ways of knowing an Archeological excavation. In: Ethnographies of Arqueological Practices. Edited by Matt Edgeworth. Lanham: Altamira Press, 2006, p. 20-32

Recebido em: 06-02-2014.

Aprovado em: 08-03-2014. 